\title{
Study on Mechanisms of Photon-Induced Material Removal on Silicon at Atomic and Close-to-Atomic Scale
}

\author{
Peizhi Wang ${ }^{1} \cdot$ Jinshi Wang ${ }^{2} \cdot$ Fengzhou Fang $^{1,2}$
}

Received: 8 July 2021 / Revised: 8 September 2021 / Accepted: 9 September 2021 / Published online: 27 October 2021

(c) The Author(s) 2021

\begin{abstract}
This paper presents a new approach for material removal on silicon at atomic and close-to-atomic scale assisted by photons. The corresponding mechanisms are also investigated. The proposed approach consists of two sequential steps: surface modification and photon irradiation. The back bonds of silicon atoms are first weakened by the chemisorption of chlorine and then broken by photon energy, leading to the desorption of chlorinated silicon. The mechanisms of photon-induced desorption of chlorinated silicon, i.e., $\mathrm{SiCl}_{2}$ and $\mathrm{SiCl}$, are explained by two models: the Menzel-Gomer-Redhead (MGR) and Antoniewicz models. The desorption probability associated with the two models is numerically calculated by solving the Liouville-von Neumann equations for open quantum systems. The calculation accuracy is verified by comparison with the results in literatures in the case of the NO/Pt (111) system. The calculation method is then applied to the cases of $\mathrm{SiCl}_{2} / \mathrm{Si}$ and $\mathrm{SiCl} / \mathrm{Si}$ systems. The results show that the value of desorption probability first increases dramatically and then saturates to a stable value within hundreds of femtoseconds after excitation. The desorption probability shows a super-linear dependence on the lifetime of excited states.
\end{abstract}

Keywords Atomic and close-to-atomic scale manufacturing · ACSM - Surface chlorination · Photon-induced desorption · Silicon

\section{Introduction}

Manufacturing development involves three paradigms, including craft-based manufacturing, precision-controllable manufacturing, and atomic and close-to-atomic scale manufacturing (ACSM) [1,2]. As the core competence of Manufacturing III, ACSM is directly focused on the removal/addition/migration of atoms and can be applied to the fabrication of atomic and close-to-atomic scale (ACS) features. Specifically, the material removal on silicon at ACS will play a significant role in the fabrication of chips, such

Fengzhou Fang

fengzhou.fang@ucd.ie

1 Centre of Micro/Nano Manufacturing Technology (MNMT-Dublin), University College Dublin, Dublin 4, Ireland

2 State Key Laboratory of Precision Measuring Technology and Instruments, Laboratory of Micro/Nano Manufacturing Technology (MNMT), Tianjin University, Tianjin 300072, China as $2 \mathrm{D}$ transistor chips [3] and quantum chips [4, 5], in the post-Moore's law era.

Several approaches have been proposed to achieve ACSM on silicon in the past years. One of the well-known approaches is scanning tunneling microscopy (STM)/atomic force microscopy (AFM)-based manipulation of silicon atoms using specific probes [6-8]. In addition to the direct manipulation of atoms, a near-single atom layer on the $\mathrm{Si}$ (100) surface can be removed by AFM probe via shearinduced mechanochemical reactions between water and silicon [9]. Although the STM/AFM-based approach has atomic precision, the low processing efficiency limits its application in industries. Another well-known approach is atomic layer deposition (ALD) [10,11], which is used to create a conformal coating on the surface of semiconductor materials. A selected precursor is first adsorbed on the surface with a single layer, followed by a reaction step to transform the precursor into a coating layer. The sequential self-limiting steps ensure the deposition of the mono-atomic layer. However, ALD is generally applied to large-area deposition and is difficult to use in the fabrication of ACS features. Similar to ALD, thermal atomic layer etching (ALE) $[12,13]$ and 
plasma-assisted ALE [14-18] were proposed to remove silicon atoms layer by layer, in which heat or plasma was used to remove the atoms modified by precursors. Nevertheless, the spatial distributions of temperature and plasma energy are difficult to control with high precision. Therefore, the two ALE approaches fail to fabricate ACS features.

Compared with heat and plasma, photons have the advantages of high spatial resolution and highly controlled scale of energy due to their unique characteristics, such as good monochromaticity and short wavelength [19-21]. Therefore, photons are a kind of potential energy for ACSM technology in the fabrication of ACS features. Initially, photons were used to assist the etching of silicon; the mechanism of this process can be divided into thermal effects at high laser fluences and non-thermal effects at low laser fluences [22, 23]. Non-thermal effects consist of the photo-dissociation of chlorine/fluorine and carrier-enhanced chemical reaction. The reaction of photo-assisted etching is not self-limiting and therefore cannot achieve atomic or close-to-atomic precision. Several attempts were performed to apply photon irradiation to the ALE of GaAs, and a near-atomic layer precision was obtained $[24,25]$. The corresponding mechanism was explained by the excitation of the $\mathrm{GaCl}$-like layer to the anti-bonding state. However, a detailed calculation based on quantum mechanics was lacking, and the application to silicon was considerably difficult because the desorption energies of chlorinated silicon are notably higher than those of the chlorinated species of GaAs [26]. Photon-induced desorption experiments with photons at different wavelengths of 245,290 , and $532 \mathrm{~nm}$ showed that $\mathrm{SiCl}_{2}$ disappeared from the chlorinated silicon surface within the irradiation region, whereas $\mathrm{SiCl}$ remained on the surface and was stable against irradiation [27, 28]. It is believed that $\mathrm{SiCl}$ can be desorbed as long as a suitable wavelength of photons is selected to break the back bonds of chlorinated silicon, and this condition highly relies on the understanding of fundamental mechanisms during the corresponding process.

In this paper, the process of photon-based ACSM on silicon is first introduced. The mechanisms of photon-induced desorption in this process are studied using two desorption models. The desorption probability associated with the two models is then numerically calculated based on quantum mechanics. Finally, the calculation accuracy is verified, and the corresponding results are presented.

\section{Photon-based ACSM Approach}

The most important aspect in the removal of target atoms without damaging bulk silicon is to ensure the self-limitation of reactions. Self-limitation implies that the reactions would stop once the target atoms are removed despite the photon irradiation of silicon. An effective way to ensure this self-limitation is a surface modification, in which the back bonds of surface atoms are weakened by specific precursors without affecting bulk silicon atoms. The weakened bonds will then be broken by photon energy, and the target atoms within the irradiation region will be subsequently removed. The underlying bulk silicon atoms will not be removed despite being exposed to photon irradiation as long as the wavelength of photons breaks the weakened back bonds but not the other bonds.

Chlorine $(\mathrm{Cl})$ can react as a precursor to modify the silicon surface by chemisorption $[14,15]$, in which chlorine gas spontaneously chemisorbs on the silicon surface and forms a monolayer of chlorinated silicon (mostly $\mathrm{SiCl}_{2}$ and $\mathrm{SiCl}$ ) instead of $\mathrm{SiCl}_{4}[29,30]$. The chemisorbed $\mathrm{Cl}$ atom weakens the back bonds of silicon atoms and decreases the desorption energies of chlorinated silicon (Fig. 1). The desorption energies of $\mathrm{SiCl}_{2}$ and $\mathrm{SiCl}$ decrease from 7.40-8.46 eV to $1.4-3.2 \mathrm{eV}$ and $4.52-5.74 \mathrm{eV}$, respectively, compared with bared silicon atoms [26, 31-36]. This condition provides a favorable surface for the next step, which is photon irradiation.

Based on the above analysis, the designed photon-based ACSM approach is divided into a sequence of self-limited reactions (Fig. 2). The silicon atoms on the surface are modified by chlorine as a precursor forming a monolayer of chlorinated silicon, followed by photon irradiation to remove the modified silicon atoms. The required structures can be obtained by repeating the sequential cycle. In the irradiation step, the modified silicon atoms are removed in the form of $\mathrm{SiCl}_{2}$ and $\mathrm{SiCl}$ as a result of photon-induced desorption. The corresponding mechanisms will be discussed in the next section.

\section{Description of Models for Photon-Induced Desorption}

Photon-induced desorption refers to the reactions in which atoms/molecules are desorbed as neutrals or ions due to electronic excitation stimulated by photons. Several models have been proposed to describe the mechanisms of photoninduced desorption; these models include the Knotek-Feibelman (KF) [37, 38], Menzel-Gomer-Redhead (MGR) $[39,40]$, and Antoniewicz model [41, 42]. The KF model is based on the ionization of a core level, followed by an Auger decay. The Auger process produces a two-hole state, leading to the desorption of absorbates as ions. The ionization of a core level requires photons with high energy; that is, it exceeds the threshold for the direct desorption of chlorine atoms [43]. In addition, experimental results show that the desorption products from silicon surfaces are mostly $\mathrm{SiCl}_{2}$ and $\mathrm{SiCl}$ rather than ions [27, 28]; therefore, the $\mathrm{KF}$ model cannot be applied to the systems of $\mathrm{SiCl}_{2} / \mathrm{Si}$ and $\mathrm{SiCl} / \mathrm{Si}$. 
Fig. 1 Desorption energies of bared $\mathrm{Si}, \mathrm{SiCl}_{2}$, and $\mathrm{SiCl}$ on the silicon surface

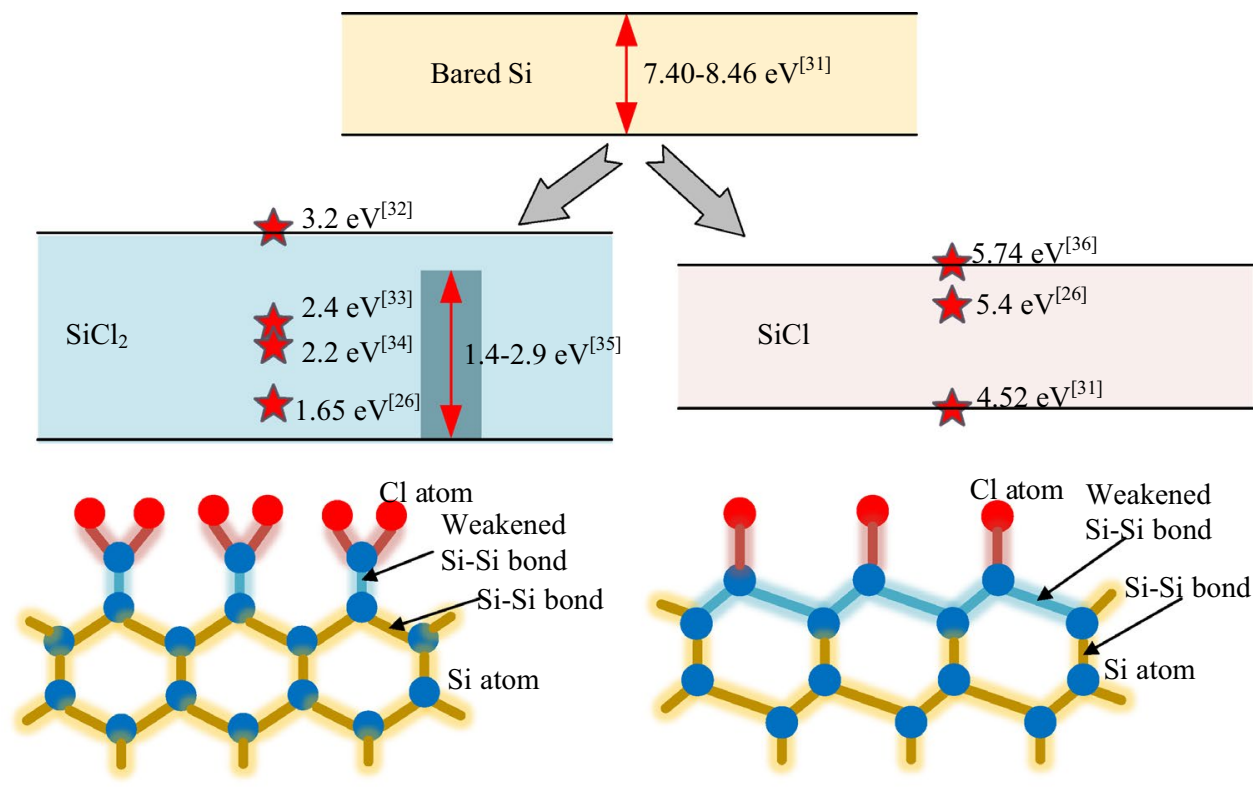

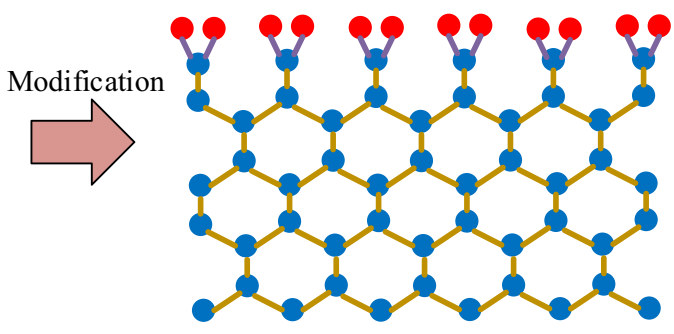

Rep
- Si atom
Cl atom
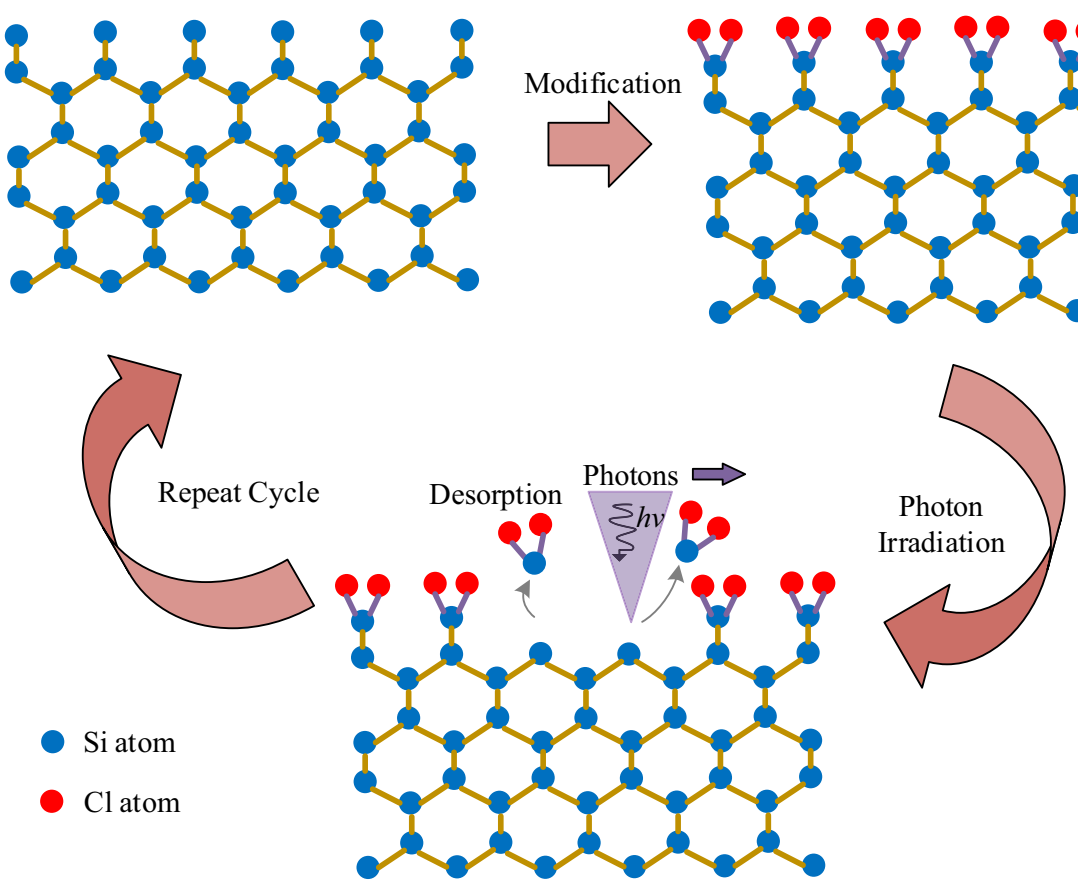

Fig. 2 Schematic of photonbased ACSM process on silicon
The mechanisms of photon-induced desorption for $\mathrm{SiCl}_{2} /$ $\mathrm{Si}$ and $\mathrm{SiCl} / \mathrm{Si}$ systems can be described by the MGR and Antoniewicz models, respectively (Figs. 3 and 4). For the $\mathrm{SiCl}_{2} / \mathrm{Si}$ system, the electrons on the back bonds of silicon are excited from the ground state to the anti-bonding state ( $\pi^{*}$ state for silicon [44]) after the absorption of photons. The excited adsorbate will then move away from the surface due to the associated repulsive potential effect (anti-bonding orbital shown in Fig. 3) before it quenches to the ground state. At the time of quenching, if the acquired kinetic energy $K_{\mathrm{E}}$ of the adsorbate exceeds the remaining potential barrier $V_{\mathrm{B}}$, then it will continue to move and desorb from the surface.

For the $\mathrm{SiCl} / \mathrm{Si}$ system, the desorption of $\mathrm{SiCl}$ cannot be explained by the MGR model given that the desorption energy of $\mathrm{SiCl}$ is notably higher than that of $\mathrm{SiCl}_{2}$ (Fig. 1), and $\mathrm{SiCl}$ experiences difficulty in obtaining sufficient kinetic energy to overcome the potential barrier according to MGR model. Here, the Antoniewicz model can be used to explain the desorption of $\mathrm{SiCl}$, in which the adsorbate is first excited to the ionized state by high-energy photons and then drops to the ground state 


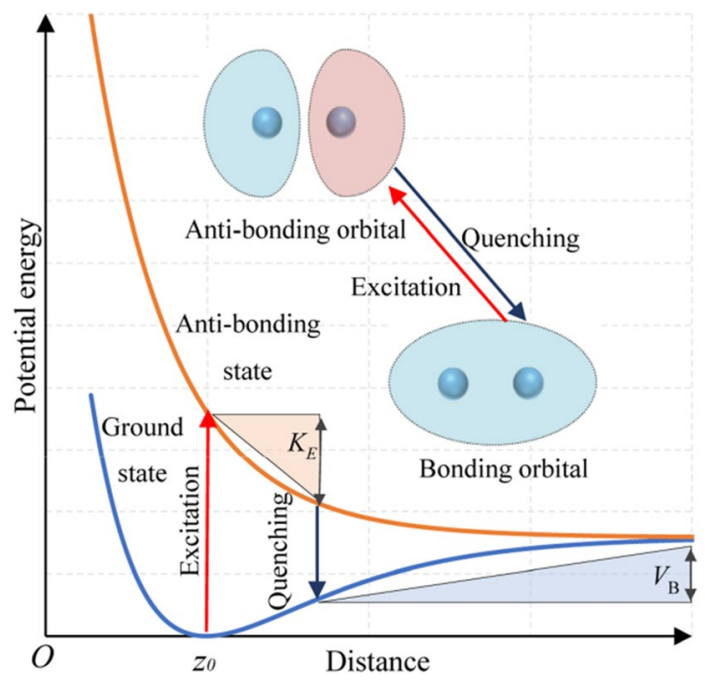

Fig. 3 Description of MGR model for photon-induced desorption of $\mathrm{SiCl}_{2} . K_{\mathrm{E}}$ is the kinetic energy, and $V_{\mathrm{B}}$ is the potential barrier at the point of quenching

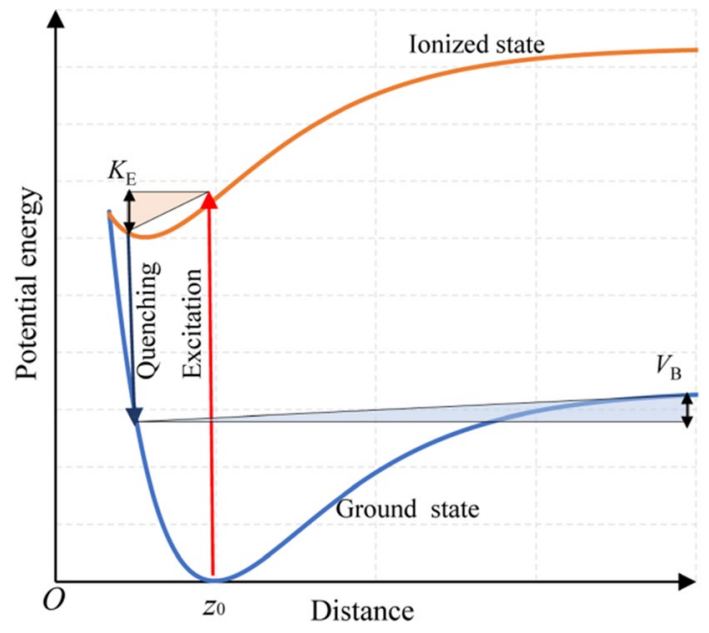

Fig. 4 Description of Antoniewicz model for photon-induced desorption of $\mathrm{SiCl}$

(Fig. 4). The adsorbate will be desorbed from the surface if the acquired kinetic energy $K_{\mathrm{E}}$ exceeds the potential barrier $V_{\mathrm{B}}$. The excitation of the ionized state requires photon energy exceeding the first ionization energy of silicon, i.e., $8.15 \mathrm{eV} \mathrm{[45],} \mathrm{and} \mathrm{this} \mathrm{condition} \mathrm{agrees} \mathrm{with}$ the results showing the stability of $\mathrm{SiCl}$ against the irradiation of photons at the wavelengths of 245, 290, and $532 \mathrm{~nm}[27,28]$. Photon energy should be below $17 \mathrm{eV}$ to avoid the direct desorption of $\mathrm{Cl}$, in which an electron from $\mathrm{Cl} 3 \mathrm{~s}$ orbital is excited, and an Auger-like process follows [43].

\section{Numerical Calculation}

As described, the excited state in the MGR and Antoniewicz models is unstable and will quench to the ground state at a certain quenching rate. The adsorbates can desorb from the surface if the acquired kinetic energy exceeds the potential barrier at the time of quenching. Otherwise, the adsorbates will remain on the surface. In this section, the desorption probability after the excitation associated with the two models is numerically calculated based on quantum mechanics for open systems. Figure 5 shows the configurations of the desorption system. After the absorption of photons, the ground state is stimulated to the excited state, i.e., anti-bonding state or ionized state, at time $t=0$. Then, the excited adsorbates evolve and move away from the surface along an angle $\theta$. In this study, the desorption angle $\theta$ is set to zero because most of the adsorbates desorb along the normal direction of the surface at high coverage of chlorine [46]. In this manner, the problem can be reduced to the calculation of desorption probability in a two-state system with one dimension.

Instead of Newton mechanics, quantum mechanics for open systems is used to obtain accurate solutions. The desorption

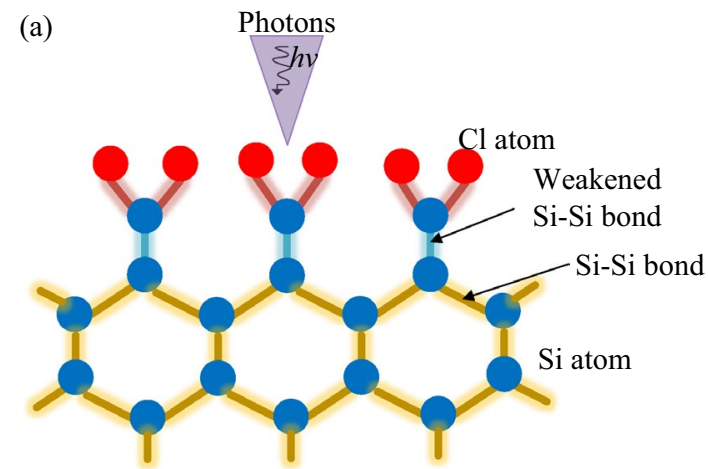

(b)

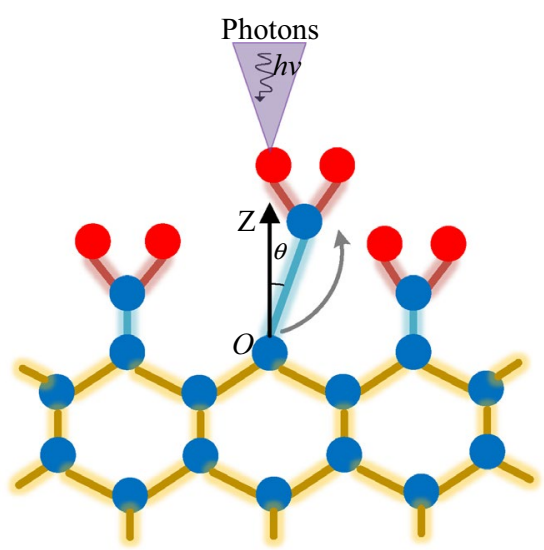

Fig. 5 Configurations of the desorption system: a $t=0$, time at the beginning of desorption; $\mathbf{b} t=t_{1}$, time after excitation 
probability can be obtained by solving the Liouville-von Neumann equation [47-49]:

$\frac{\partial \hat{\rho}}{\partial t}=\mathcal{L} \hat{\rho}=\mathcal{L}_{\mathrm{H}} \hat{\rho}+\mathcal{L}_{\mathrm{D}} \hat{\rho}$

where $\hat{\rho}$ is the density operator, $t$ is the evolution time, $\mathcal{L}$ is the total Liouvillian operator, $\mathcal{L}_{\mathrm{H}}$ and $\mathcal{L}_{\mathrm{D}}$ are Hamiltonian Liouvillian operator and dissipative Liouvillian operator, respectively.

$\mathcal{L}_{\mathrm{H}} \hat{\rho}=-\frac{i}{\hbar}[\hat{H}, \hat{\rho}]$

$\mathcal{L}_{\mathrm{D}} \hat{\rho}=\sum_{k=1}^{K}\left(\hat{C}_{k} \hat{\rho} \hat{C}_{k}^{+}-\frac{1}{2}\left[\hat{C}_{k}^{+} \hat{C}_{k}, \hat{\rho}\right]_{+}\right)$

where $[A, B]$ and $[A, B]_{+}$denote the commutator and anticommutator, respectively, $\hbar$ is the reduced Planck's constant, $\hat{H}$ is the system Hamiltonian operator, $\hat{C}_{k}$ is the Lindblad operator through the dissipative channel $k$, and $k=1$ represents the spontaneous decay from the excited state to the ground state,

$\hat{H}=\hat{H}_{e}|e\rangle\left\langle e\left|+\hat{H}_{g}\right| g\right\rangle\left\langle g\left|+\hat{V}_{e g}\right| e\right\rangle\left\langle g\left|+\hat{V}_{g e}\right| g\right\rangle\langle e|$

$\hat{C}_{1}=\sqrt{\Gamma_{g e}}|g\rangle\langle e|$

$\hat{\rho}=\hat{\rho}_{e e}|e\rangle\left\langle e\left|+\hat{\rho}_{g g}\right| g\right\rangle\left\langle g\left|+\hat{\rho}_{e g}\right| e\right\rangle\left\langle g\left|+\hat{\rho}_{g e}\right| g\right\rangle\langle e|$

where $\hat{V}_{e g}$ and $\hat{V}_{g e}$ are the Hamiltonian coupling operators, $|g\rangle$ and $|e\rangle$ represent the ground and excited states, respectively, $\hat{\rho}_{m n}=\langle m|\hat{\rho}| n\rangle(m, n=g, e), \Gamma_{g e}$ is the quenching rate, $\Gamma_{g e}=1 / \tau, \tau$ is the lifetime of the excited state, and $\hat{H}_{l}(l=g$, $e$ ) can be represented as follows:

$\hat{H}_{l}=\hat{K}+\hat{V}_{l}(Z)=-\frac{\hbar^{2}}{2 m} \frac{\partial^{2}}{\partial Z^{2}}+\hat{V}_{l}(Z), l=g, e$

where $m$ represents the molecular mass of the adsorbate, and $\hat{V}_{l}(l=g, e)$ represents the potential energy operators for the ground and excited states.

After obtaining the density operator $\hat{\rho}$ in accordance with the algorithm in Fig. 6, the desorption probability at any time $t$ can be calculated by the following:

$P_{d}(t)=\operatorname{tr}\left\{\hat{\theta}\left(Z-Z_{d}\right) \hat{\rho}(t)\right\}$

where

$\hat{\theta}\left(Z-Z_{d}\right)= \begin{cases}0, & Z \leq Z_{d} \\ 1, & Z>Z_{d}\end{cases}$

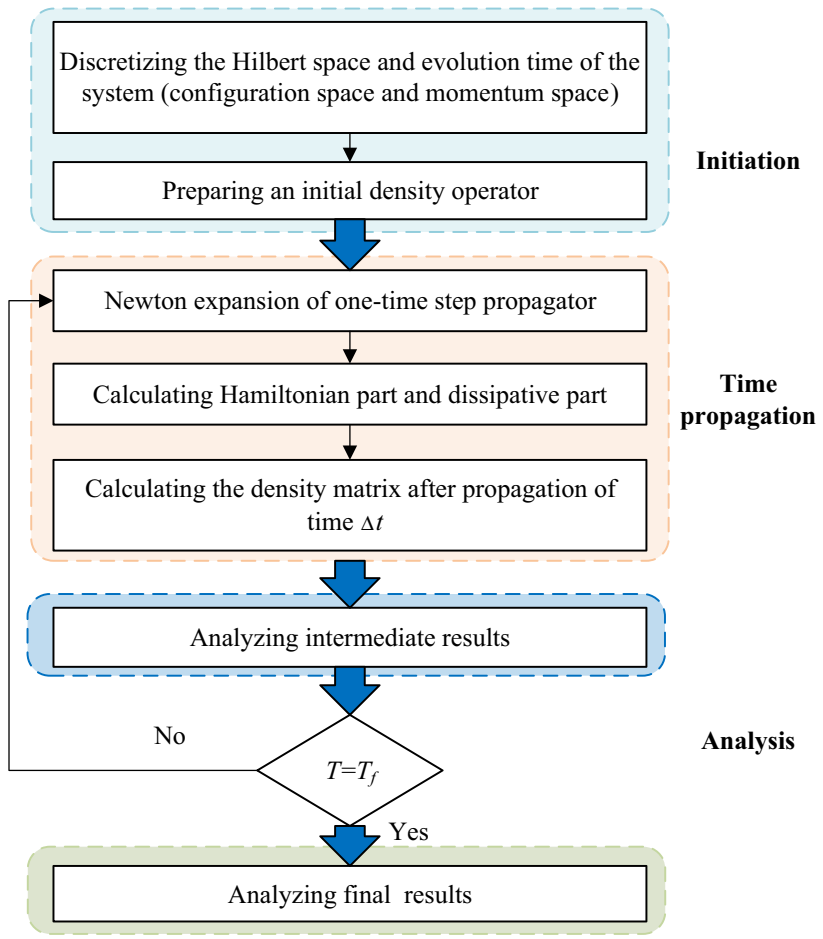

Fig. 6 Algorithm for the numerical calculation process, which is divided into three parts: initiation, time propagation, and analysis

where $Z_{d}$ is the critical distance beyond which the adsorbate is considered to have been desorbed from the surface.

The algorithm in Fig. 6 includes initiation, time propagation, and analysis parts. The density operator in the coordinate representation can be calculated through the initiation and time propagation parts, as will be discussed in detail. Then, the desorption probability is calculated by Eqs. (8) and (9). Here, direct matrix manipulation and a new routine for the determination of uniformly distributed sampling points are applied to the time propagation part to improve the calculation efficiency.

In the initiation part, the Hilbert space of the system, i.e., the coordinate and momentum spaces, can be discretized by the following:

$Z_{i}=Z_{0}+(i-1) \Delta Z, i=1,2, \ldots, N$

$\Delta k=\frac{2 \pi}{(N-1) \Delta Z}$

$k_{i}=-\frac{\pi}{\Delta Z}+(i-1) \Delta k, i=1,2, \ldots, N$

where $N$ is the total number of discretizing points.

The initial density operator at time $t=0$ has the following form: 
$\hat{\rho}(0)=\hat{\sigma}|e\rangle\langle e|$

where $\hat{\sigma}$ is a ground-state density operator and can be written as follows:

$\hat{\sigma}=\sum_{v} w_{v}\left|v_{g}\right\rangle\left\langle v_{g}\right|$

$w_{v}=\delta_{v v^{\prime}}$

where $\left|v_{g}\right\rangle$ is the vibrational state of ground potential operator $\hat{V}_{l}$, i.e., eigenfunctions of $\hat{H}_{g}$. Here, $w_{v}=\delta_{v 0}, \delta$ is the Kronecker delta function:

$\hat{H}_{g}\left|v_{g}\right\rangle=\varepsilon_{v g}\left|v_{g}\right\rangle$

The discretizing form of $\left|v_{g}\right\rangle$ on coordinate representation can be obtained using the Fourier grid Hamiltonian method [50].

In the time propagation part, the density operator after one-time step $(\Delta t)$ of propagation can be calculated [47] by the following:

$\hat{\rho}(t+\Delta t)=\mathrm{e}^{\mathcal{L} \Delta t} \hat{\rho}(t)$

The time propagator $\mathrm{e}^{\mathcal{L} \Delta t}$ in Eq. (17) can be expressed by Newton polynomial of order $M$ :

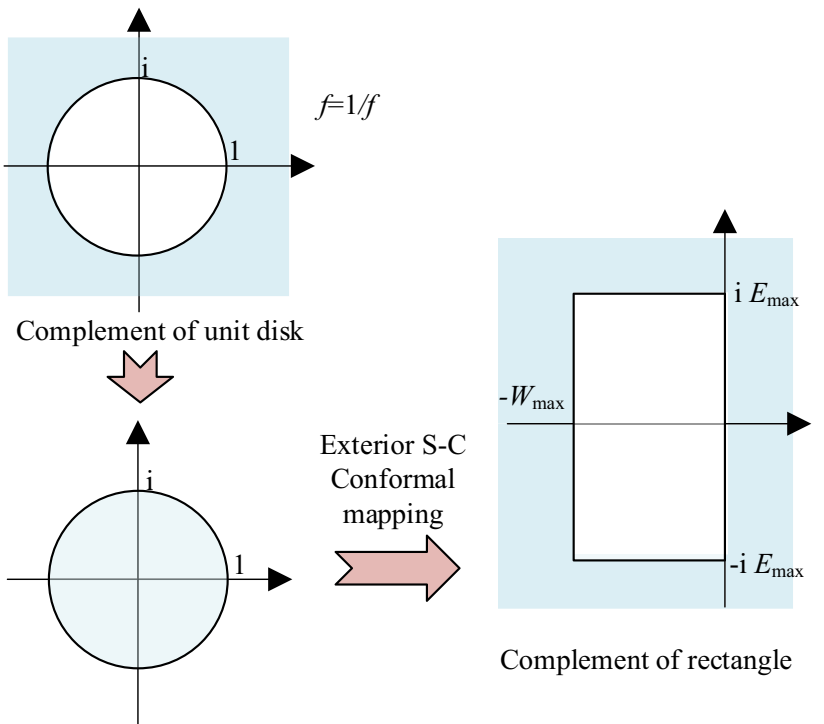

Interior of unit disk

Fig. 7 Routine for the determination of uniformly distributed sampling point $z_{i}$

into the complement of a rectangle by exterior S-C conformal mapping [53].

Once the Newton polynomial expansion of the time propagator in Eq. (18) has been obtained, the density operator

$\mathrm{e}^{\mathcal{L} \Delta t} \approx c_{0} \mathcal{J}+c_{1}\left(\mathcal{L}-z_{0} \mathcal{J}\right)+c_{2}\left(\mathcal{L}-z_{1} \mathcal{J}\right)\left(\mathcal{L}-z_{0} \mathcal{J}\right)+\cdots+c_{M}\left(\mathcal{L}-z_{M-1} \mathcal{J}\right) \cdots\left(\mathcal{L}-z_{1} \mathcal{J}\right)\left(\mathcal{L}-z_{0} \mathcal{J}\right)$

where $c_{i}(i=0,1, \ldots, M)$ are the difference coefficients, $\mathcal{J}$ is an identity operator, $z_{i}$ is the uniformly distributed sampling point on the boundary of a complex rectangle $\{(0$, $\left.\left.-\mathrm{i} E_{\max }\right),\left(0, \mathrm{i} E_{\max }\right),\left(-W_{\max }, \mathrm{i} E_{\max }\right),\left(-W_{\max },-\mathrm{i} E_{\max }\right)\right\}$, $W_{\max }$ is the maximum eigenvalue of the operator $\hat{C}_{k} \hat{C}_{k}^{+}$, and $E_{\max }$ is the maximum Hamiltonian energy and can be evaluated as follows:

$E_{\max } \approx V_{\max }-V_{\text {min }}+k_{\max }^{2} / 2 m$

with $k_{\max }$ representing the maximum momentum on the discretizing grids.

The uniformly distributed sampling point $z_{i}$ can be obtained based on a Schwarz-Christoffel (S-C) conformal mapping method (Fig. 7). If a conformal mapping function $f_{m}$ that can transform the complement of unit disk to the complement of a rectangle exists, then the points $f_{m}\left(c r_{i}\right)(i=0,1, \ldots, M-1)$ will be the required points where $c r_{i}(i=0,1, \ldots, M-1)$ are the uniformly distributed points on the unit circle [51]. The $M-1$ points of $c r_{i}$ are selected based on the algorithm proposed in the literature [52]. Here, the complement of the unit disk will be first transformed into the unit disk interior and then transformed again at any time can be calculated by replacing Eqs. (18) and (1) into Eq. (17). For the completion of this part, the action of $\mathcal{L}_{\mathrm{H}}$ and $\mathcal{L}_{\mathrm{D}}$ on $\hat{\rho}(t)$ can be directly calculated in the form of a matrix due to the powerful capability of MATLAB to deal with matrixes.

$\mathcal{L}_{\mathrm{H}} \hat{\rho}=-\frac{i}{\hbar}\left[\left(\begin{array}{cc}\hat{H}_{e} & \hat{V}_{e g} \\ \hat{V}_{g e} & \hat{H}_{g}\end{array}\right),\left(\begin{array}{cc}\hat{\rho}_{e e} & \hat{\rho}_{e g} \\ \hat{\rho}_{g e} & \hat{\rho}_{g g}\end{array}\right)\right]$

$\mathcal{L}_{\mathrm{D}} \hat{\rho}=\hat{\Gamma}_{g e}\left(\begin{array}{cc}-\hat{\rho}_{e e} & -\frac{1}{2} \hat{\rho}_{e g} \\ -\frac{1}{2} \hat{\rho}_{g e} & \hat{\rho}_{e e}\end{array}\right)$

\section{Results and Discussion}

In this section, the calculation accuracy of the numerical method is first verified by comparison with the results in literature in the case of the NO/Pt (111) system. Then, the desorption probabilities in the cases of $\mathrm{SiCl}_{2} / \mathrm{Si}$ and $\mathrm{SiCl} / \mathrm{Si}$ are calculated and discussed. 


\subsection{Accuracy Verification}

The reason for selecting the NO/Pt (111) system is that the system is well studied for desorption, and the related data of desorption probability can be easily obtained. Figure 8 shows a comparison of the desorption probability between the numerical calculation results and those in the literature [54]. The same potential, initiation, and propagation parameters $(\tau=4 \mathrm{fs})$ in the literature are used for numerical calculation. The two curves have similar trends along the propagation time, and the relative error of the final values of desorption probability is $5.07 \%$.

\subsection{Case Studies}

\subsection{1 $\mathrm{SiCl}_{2} / \mathrm{Si}$ System}

The desorption probabilities in the two cases described in Sect. 3 are calculated based on the numerical calculation method. The Hamiltonian coupling operators $\hat{V}_{e g}$ and $\hat{V}_{g e}$ are neglected. The ground potentials for $\mathrm{SiCl}_{2} / \mathrm{Si}$ and $\mathrm{SiCl} / \mathrm{Si}$ in Eq. (7) can be described by a modified Tersoff function [55]:

$V_{g}(Z)=f_{i} f_{c}\left(Z / \cos \frac{\phi}{2}\right)\left[A \mathrm{e}^{\left(-\lambda Z / \cos \frac{\phi}{2}\right)}-B \mathrm{e}^{\left(-\mu Z / \cos \frac{\phi}{2}\right)}\right]$

where $f_{i}(i=1,2)$ is the modified coefficient describing the effect of chlorine, with $i=1$ for $\mathrm{SiCl}_{2}$ and $i=2$ for $\mathrm{SiCl}, \phi$ is the bonding angle of $\mathrm{Si}-\mathrm{Si}$, and $f_{\mathrm{c}}$ is the cut-off function to improve the calculation efficiency.

The anti-bonding potential for $\mathrm{SiCl}_{2} / \mathrm{Si}$ system can be estimated as the follows [56]:

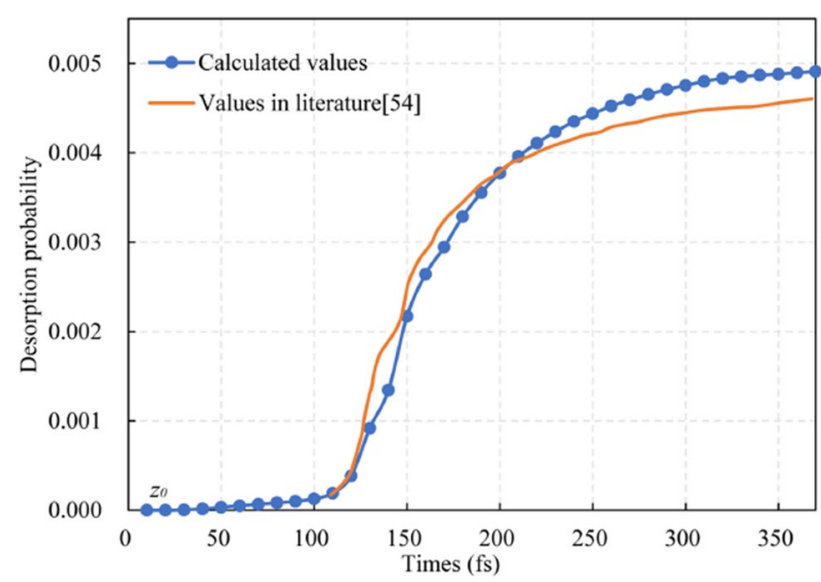

Fig. 8 Comparison of the desorption probability between numerical calculation results and findings in literature for the NO/Pt(111) system
$V_{\mathrm{e}}(Z)=f_{\mathrm{e}}\left[\mathrm{e}^{\left(-\lambda\left(Z / \cos \frac{\phi}{2}-Z_{\mathrm{b}}\right)\right)}+\mathrm{e}^{\left(-\mu\left(Z / \cos \frac{\phi}{2}-Z_{\mathrm{b}}\right)\right)}\right]$

Table 1 shows the potential, initiation, and propagation parameters used in the calculation. Figure 9 displays the snapshots of the density in the ground and excited states along the separation distance for the $\mathrm{SiCl}_{2} / \mathrm{Si}$ system. The lifetime of the excited state $\tau$ is assumed to be $2 \mathrm{fs}$. The ground state is excited to the anti-bonding state by Franck-Condon transition at time $t=0$. With time propagation, the excited state gradually drops to the ground state, where the density peak of the excited state declines, and the peak of the ground state rises.

Figure 10 shows the desorption probability of $\mathrm{SiCl}_{2}$ as a function of propagation time. When the propagation time

Table 1 Calculation parameters for the $\mathrm{SiCl}_{2} / \mathrm{Si}$ and $\mathrm{SiCl} / \mathrm{Si}$ systems

Potential parameters $[31,35,45,55,56]$

\begin{tabular}{llll}
\hline$f_{1}$ & 0.8064 & $f_{2}$ & 1.6954 \\
$A$ & $1830.8 \mathrm{eV}$ & $B$ & $471.2 \mathrm{eV}$ \\
$\phi$ & $109^{\circ}$ & $\mu$ & $1.7322 \AA^{-1}$ \\
$\lambda$ & $2.4799 \AA^{-1}$ & $f_{\mathrm{e}}$ & $1.32 \mathrm{eV}$ \\
$\mathrm{Z}_{\mathrm{b}}$ & $2.318 \AA$ & $Z_{\mathrm{e}}$ & $0.4 \AA$ \\
$V_{\text {ion }}$ & $8.15 \mathrm{eV}$ & & \\
Initiation parameters & & \\
$Z_{0}$ & $1.6 a_{0}$ & $N$ & 512 \\
$\Delta Z$ & $0.018 a_{0}$ & & \\
Propagation parameters & & \\
$\Delta t$ & $0.5 \mathrm{fs}$ & $T_{f}$ & $400 \mathrm{fs} / 200 \mathrm{fs}$ \\
$M$ & 32 & $\tau$ & $1-4 \mathrm{fs}$ \\
$Z_{\mathrm{d}}$ & $6.0 a_{0}$ & & \\
\hline
\end{tabular}

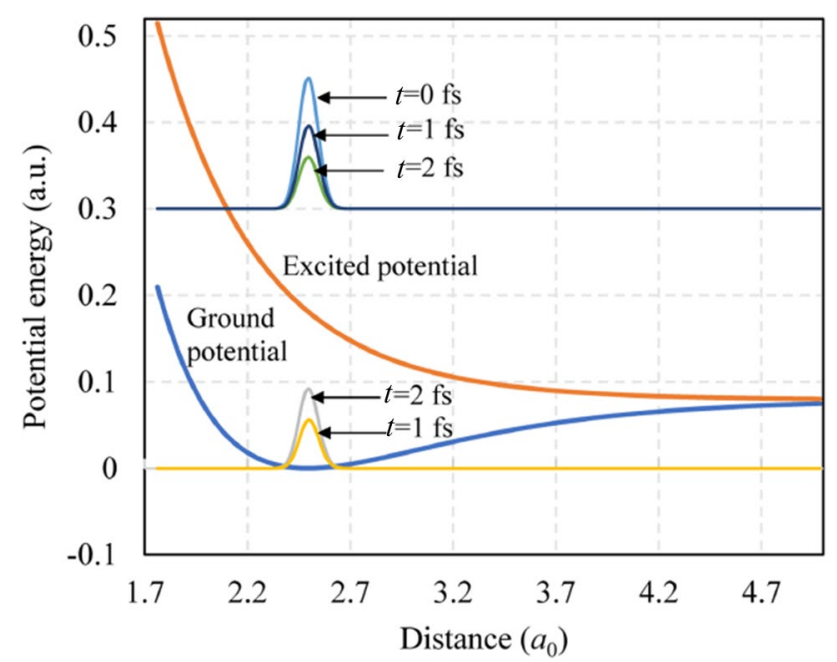

Fig. 9 Snapshots of the density in the ground and excited states along the separation distance for the $\mathrm{SiCl}_{2} / \mathrm{Si}$ system. $\tau=2 \mathrm{fs}$ and $t=0$ is the time at the beginning of desorption 


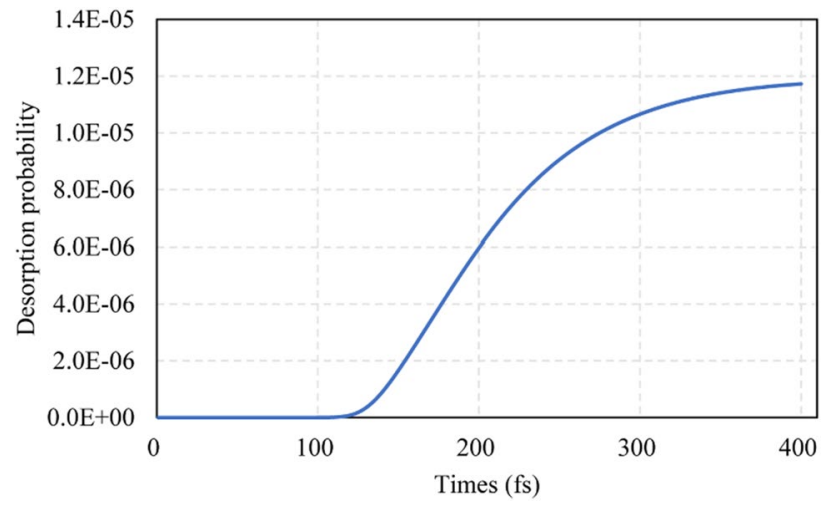

Fig. 10 Desorption probability of $\mathrm{SiCl}_{2}$ as a function of propagation time for the $\mathrm{SiCl}_{2} / \mathrm{Si}$ system, $\tau=2 \mathrm{fs}$

exceeds a critical value, the desorption probability increases dramatically and then gradually saturates to a stable value, which is the final value of desorption probability. The propagation time to reach stable values is within hundreds of femtoseconds, which means that the total reaction time for desorption is on the sub-picosecond scale.

Figure 11 shows the stable value of desorption probability as a function of the lifetime of excited state $\tau$. The desorption probability exhibits a super-linear dependence on $\tau$. If the lifetime of the excited state can be increased, then the manufacturing efficiency of the photon-based ACSM approach will be improved significantly.

\subsubsection{SiCl/Si System}

The ionized-state potential for $\mathrm{SiCl} / \mathrm{Si}$ system has the following form based on the ionized potential in literature [54]; the corresponding parameters are shown in Table 1.

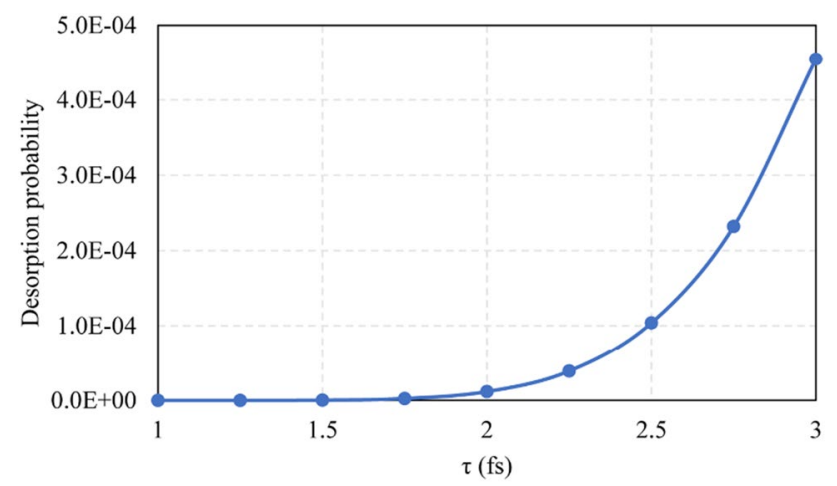

Fig. 11 Desorption probability of $\mathrm{SiCl}_{2}$ as a function of excited state lifetime $\tau$ for the $\mathrm{SiCl}_{2} / \mathrm{Si}$ system

$$
\begin{aligned}
& V_{\mathrm{e}}(Z)=f_{2} f_{\mathrm{c}}\left(Z / \cos \frac{\phi}{2}+Z_{\mathrm{e}}\right) \\
& {\left[A \mathrm{e}^{\left(-\lambda Z / \cos \frac{\phi}{2}+Z_{\mathrm{e}}\right)}-B \mathrm{e}^{\left(-\mu Z / \cos \frac{\phi}{2}+Z_{\mathrm{e}}\right)}\right]+V_{\text {ion }}}
\end{aligned}
$$

Figure 12 shows the desorption probability of $\mathrm{SiCl}$ as a function of propagation time. This curve has a similar trend to the curve in Fig. 10, but the value is slightly lower. The lower value means that the desorption efficiency of $\mathrm{SiCl}$ is lower than that of $\mathrm{SiCl}_{2}$, and more efficient methods should be proposed to increase the efficiency. In addition, less time is required for $\mathrm{SiCl}$ to reach the saturated value than $\mathrm{SiCl}_{2}$ because $\mathrm{SiCl}$ has a smaller molecular mass.

This paper mainly investigates the mechanisms of photon-induced material removal on silicon, and experimental implementation of the proposed approach still needs to be further investigated. Although this approach is promising, the following challenges will have to be overcome, i.e., the preparation of atomic-scale flattening silicon surface, the integration of setups, and metrology. The atomic-scale flattening silicon surface is the target surface where the experiments will be carried out, and it can be obtained by wetting treatment with $\mathrm{NH}_{4} \mathrm{~F}$ solutions. The approach requires a special-designed laser with a short wavelength, and the setups should be integrated into an ultra-high vacuum system, which is challenging. Furthermore, metrology requires atomic-scale precision and online measurements. Therefore, STM/AFM may need to be integrated into the system.

\section{Conclusions}

A new approach for the photon-induced material removal on silicon at ACS is developed, and the corresponding mechanisms are investigated. The main conclusions from this study can be drawn as follows.

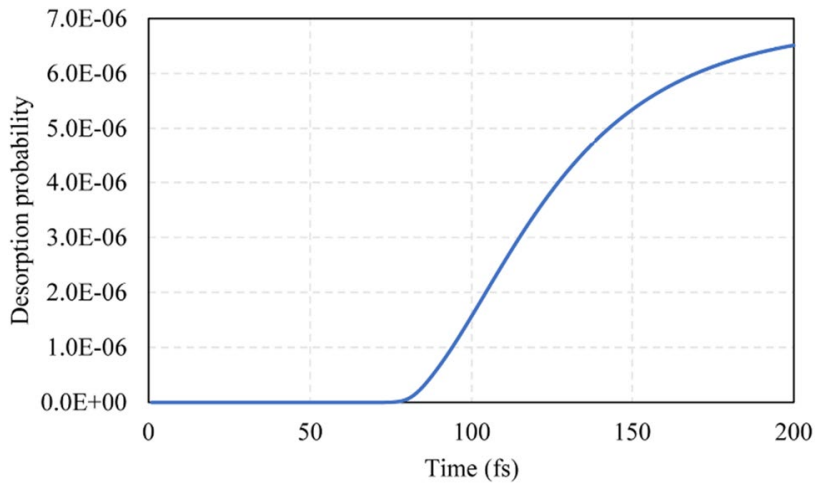

Fig. 12 Desorption probability of $\mathrm{SiCl}$ as a function of propagation time for the $\mathrm{SiCl} / \mathrm{Si}$ system, $\tau=2 \mathrm{fs}$ 
(1) A new approach for ACSM on silicon is proposed by using chlorine to modify the silicon atoms on the surface and then using photon irradiation to desorb the modified silicon atoms in the form of $\mathrm{SiCl}_{2} / \mathrm{SiCl}$.

(2) Mechanisms of the photon-induced desorption of $\mathrm{SiCl}_{2}$ and $\mathrm{SiCl}$ from the silicon surface are studied by the MGR model, corresponding to the anti-bonding state, and the Antoniewicz model, corresponding to the ionized state. The desorption probability associated with the two models is numerically calculated based on quantum mechanics. The calculation accuracy is verified by comparing with the results in literature in the case of NO/Pt (111) system with the relative error of $5.07 \%$.

(3) The values of desorption probability first increase dramatically and then saturate to stable values with propagation time within hundreds of femtoseconds. The results also show the super-linear dependence of desorption probability on the lifetime of the excited state, which indicates that the increase in this lifetime will help in increasing the manufacturing efficiency significantly.

Acknowledgements This work was supported by the Science Foundation Ireland (SFI) (No.15/RP/B3208) and the National Natural Science Foundation of China (NSFC) (No. 52035009). The authors would also like to thank Marco Castelli, Zhichao Geng, Chenghao Chen, and Jintong $\mathrm{Wu}$ for valuable discussions.

\section{Declarations}

Conflict of interest The authors declare no conflicts of interest.

Open Access This article is licensed under a Creative Commons Attribution 4.0 International License, which permits use, sharing, adaptation, distribution and reproduction in any medium or format, as long as you give appropriate credit to the original author(s) and the source, provide a link to the Creative Commons licence, and indicate if changes were made. The images or other third party material in this article are included in the article's Creative Commons licence, unless indicated otherwise in a credit line to the material. If material is not included in the article's Creative Commons licence and your intended use is not permitted by statutory regulation or exceeds the permitted use, you will need to obtain permission directly from the copyright holder. To view a copy of this licence, visit http://creativecommons.org/licenses/by/4.0/.

\section{References}

1. Fang FZ (2020) On atomic and close-to-atomic scale manufacturing-development trend of manufacturing technology. China Mech Eng 31:1009-1021

2. Fang FZ (2020) Atomic and close-to-atomic scale manufacturing: perspectives and measures. Int J Extreme Manuf 2:030201
3. Liu Y, Duan X, Shin HJ, Park S, Huang Y, Duan X (2021) Promises and prospects of two-dimensional transistors. Nature 591:43-53

4. Chen S, Li W, Wu J, Jiang Q, Tang M, Shutts S et al (2016) Electrically pumped continuous-wave III-V quantum dot lasers on silicon. Nat Photon 10:307

5. Lawrie WIL, Eenink HGJ, Hendrickx NW, Boter JM, Petit L, Amitonov SV et al (2020) Quantum dot arrays in silicon and germanium. Appl Phys Lett 116:080501

6. Eigler DM, Schweizer EK (1990) Positioning single atoms with a scanning tunnelling microscope. Nature 344:524-526

7. Sugimoto Y, Abe M, Morita S (2015) Atom manipulation using atomic force microscopy at room temperature. Imaging and manipulation of adsorbates using dynamic force microscopy. Springer, Cham, pp 49-62

8. Mathew PT, Rodriguez BJ, Fang FZ (2020) Atomic and closeto-atomic scale manufacturing: a review on atomic layer removal methods using atomic force microscopy. Nanomanuf Metrol 3:167-186

9. Chen L, Wen J, Zhang P, Yu B, Chen C, Ma T et al (2018) Nanomanufacturing of silicon surface with a single atomic layer precision via mechanochemical reactions. Nat Commun 9:1-7

10. George SM (2010) Atomic layer deposition: an overview. Chem Rev 110:111-131

11. Li S, Xu J, Wang L, Yang N, Ye X, Yuan X et al (2020) Effect of post-deposition annealing on atomic layer deposited $\mathrm{SiO}_{2}$ film for silicon surface passivation. Mat Sci Semicon Proc 106:104777

12. Abdulagatov AI, George SM (2018) Thermal atomic layer etching of silicon using $\mathrm{O}_{2}, \mathrm{HF}$, and $\mathrm{Al}\left(\mathrm{CH}_{3}\right)_{3}$ as the reactants. Chem Mater 30:8465-8475

13. Cheng PH, Wang CI, Ling CH, Lu CH, Yin YT, Chen MJ (2019) Low-temperature conformal atomic layer etching of $\mathrm{Si}$ with a damage-free surface for next-generation atomic-scale electronics. ACS Appl Nano Mater 2:4578-4583

14. Oehrlein GS, Metzler D, Li C (2015) Atomic layer etching at the tipping point: an overview. ECS J Solid State Sc 4:N5041

15. Kanarik KJ, Lill T, Hudson EA, Sriraman S, Tan S, Marks J et al (2015) Overview of atomic layer etching in the semiconductor industry. J Vac Sci Technol A 33:020802

16. Huard CM, Zhang Y, Sriraman S, Paterson A, Kanarik KJ, Kushner MJ (2017) Atomic layer etching of 3D structures in silicon: self-limiting and nonideal reactions. J Vac Sci Technol A 35:031306

17. Khan SA, Suyatin DB, Sundqvist J, Graczyk M, Junige M, Kauppinen C et al (2018) High-definition nanoimprint stamp fabrication by atomic layer etching. ACS Appl Nano Mater $1: 2476-2482$

18. Song EJ, Kim JH, Kwon JD, Kwon SH, Ahn JH (2018) Silicon atomic layer etching by two-step plasma process consisting of oxidation and modification to form (NH4) ${ }_{2} \mathrm{SiF}_{6}$, and its sublimation. Jpn J Appl Phys 57:106505

19. Ishii M, Meguro T, Gamo K, Sugano T, Aoyagi Y (1993) Digital etching using $\mathrm{KrF}$ excimer laser: approach to atomic-ordercontrolled etching by photo induced reaction. Jap J Appl Phys 32:6178

20. Moon SW, Jeon C, Hwang HN, Hwang CC, Song HJ, Shin HJ et al (2007) Nanolayer patterning based on surface modification with extreme ultraviolet light. Adv Mater 19:1321-1324

21. Guo B, Sun J, Hua Y, Zhan N, Jia J, Chu K (2020) Femtosecond laser micro/nano-manufacturing: theories, measurements, methods, and applications. Nanomanuf Metrol 3:26-67

22. Kullmer R, Bäuerle D (1987) Laser-induced chemical etching of silicon in chlorine atmosphere. Appl Phys A-Mater 43:227-232 
23. Rhodin T (1995) Photochemical desorption from chlorinated Si (100) and Si (111) surfaces-mechanisms and models. Prog Surf Sci 50:131-146

24. Meguro T, Aoyagi Y (1997) Digital etching of GaAs. Appl Surf Sci 112:55-62

25. Meguro T, Sakai K, Yamamoto Y, Sugano T, Aoyagi Y (1996) Tunable UV laser induced digital etching of GaAs: wavelength dependence of etch rate and surface processes. Appl Surf Sci 106:365-368

26. Knizikevičius R (2003) Evaluation of desorption activation energy of $\mathrm{SiCl}_{2}$ molecules. Surf Sci 531:L347-L350

27. Amasuga H, Nakamura M, Mera Y, Maeda K (2002) The atomic processes of ultraviolet laser-induced etching of chlorinated silicon (111) surface. Appl Surf Sci 197:577-580

28. Iimori T, Hattori K, Shudo K, Iwaki T, Ueta M, Komori F (1998) Laser-induced mono-atomic-layer etching on Cl-adsorbed Si (111) surfaces. Appl Surf Sci 130:90-95

29. Durbin TD, Simpson WC, Chakarian V, Shuh DK, Varekamp PR, Lo CW, Yarmoff JA (1994) Stimulated desorption of $\mathrm{Cl}^{+}$and the chemisorption of $\mathrm{Cl}_{2}$ on $\mathrm{Si}$ (111)- $7 \times 7$ and $\mathrm{Si}(100)-2 \times 1$. Surf Sci 316:257-266

30. Hattori K, Shudo K, Iimori T, Komori F, Murata Y (1996) Laserinduced desorption from silicon (111) surfaces with adsorbed chlorine atoms. J Phys-Condens Mat 8:6543

31. Smith PV, Cao PL (1995) Semiempirical calculations of the chemisorption of chlorine on the $\mathrm{Si}(111) 7 * 7$ surface. J Phys-Condens Mat 7:7125

32. De Wijs GA, De Vita A, Selloni A (1997) Mechanism for $\mathrm{SiCl}_{2}$ formation and desorption and the growth of pits in the etching of Si (100) with chlorine. Phys Rev Lett 78:4877

33. Sakurai S, Nakayama T (2002) Electronic structures and etching processes of chlorinated Si (111) surfaces. Jpn J Appl Phys 41:2171

34. Shudo K, Kirimura T, Tanaka Y, Ishikawa T, Tanaka M (2006) Quantitative analysis of thermally induced desorption during halogen-etching of a silicon (111) surface. Surf Sci 600:3147-3153

35. De Wijs GA, De Vita A, Selloni A (1998) First-principles study of chlorine adsorption and reactions on Si (100). Phys Rev B $57: 10021$

36. Halicioglu T, Srivastava D (1999) Energetics for bonding and detachment steps in etching of Si by Cl. Surf Sci 437:L773-L778

37. Knotek ML, Feibelman PJ (1978) Ion desorption by core-hole Auger decay. Phys Rev Lett 40:964

38. Tachibana T, Yamashita T, Nagira M, Yabuki H, Nagashima Y (2018) Efficient and surface site-selective ion desorption by positron annihilation. Sci Rep 8:1-7

39. Menzel D, Robert G (1964) Desorption from metal surfaces by low-energy electrons. J Chem Phys 41:3311-3328

40. Lackner M, Lucaßen D, Hasselbrink E (2018) Bimodal velocity distributions in the photodesorption of CO from $\mathrm{Si}$ (100) suggest V-to-T energy transfer. Chem Phys Lett 713:277-281
41. Antoniewicz PR (1980) Model for electron-and photon-stimulated desorption. Phys Rev B 21:3811

42. Abujarada S, Flathmann C, Koehler SP (2017) Translational and rotational energy distributions of $\mathrm{NO}$ photodesorbed from $\mathrm{Au}$ (100). J Phys Chem C 121:19922-19929

43. Yonezawa T, Daimon H, Nakatsuji K, Sakamoto K, Suga S, Namba H, Ohta T (1994) Photon-stimulated desorption mechanism of $\mathrm{Cl}^{+}$ions from $\mathrm{Cl} / \mathrm{Si}$ (111) surface. Jpn J Appl Phys 33:2248

44. Kanasaki J, Nakamura M, Ishikawa K, Tanimura K (2002) Primary processes of laser-induced selective dimer-layer removal on Si (001)-(2×1). Phys Rev Lett 89:257601

45. Lide DR (2004) CRC handbook of chemistry and physics. CRC Press, Boca Raton

46. Feil H, Baller TS, Dieleman J (1992) Effects of post-desorption collisions on the energy distribution of $\mathrm{SiCl}$ molecules pulsedlaser desorbed from Cl-covered Si surfaces: Monte-Carlo simulations compared to experiments. Appl Phys A-Mater 55:554-560

47. Saalfrank P, Kosloff R (1996) Quantum dynamics of bond breaking in a dissipative environment: indirect and direct photodesorption of neutrals from metals. J Chem Phys 105:2441-2455

48. Boendgen G, Saalfrank P (1998) STM-induced desorption of hydrogen from a silicon surface: an open-system density matrix study. J Phys Chem B 102:8029-8035

49. Saalfrank P, Baer R, Kosloff R (1994) Density matrix description of laser-induced hot electron mediated photodesorption of $\mathrm{NO}$ from Pt (111). Chem Phys Lett 230:463-472

50. Balint-Kurti GG, Ward CL, Marston CC (1991) Two computer programs for solving the Schrödinger equation for bound-state eigenvalues and eigenfunctions using the Fourier grid Hamiltonian method. Comput Phys Commun 67:285-292

51. Berman M, Kosloff R, Tal-Ezer H (1992) Solution of the timedependent Liouville-von Neumann equation: dissipative evolution. J Phys A-Math Gen 25:1283

52. Tal-Ezer H (1988) High degree interpolation polynomial in Newton form. Tech Rep :88-39 ICASE.

53. Driscoll TA (1996) Algorithm 756: a MATLAB toolbox for Schwarz-Christoffel mapping. ACM T Math Software (TOMS) 22:168-186

54. Saalfrank P (1996) Stochastic wave packet vs. direct density matrix solution of Liouville-von Neumann equations for photodesorption problems. Chem Phys 211:265-276

55. Tersoff J (1988) Empirical interatomic potential for silicon with improved elastic properties. Phys Rev B 38:9902

56. Keyes RW (1975) Bonding and antibonding potentials in group-IV semiconductors. Phys Rev Lett 34:1334 